Urologe 2006 · [Sonderheft] 45:127-133 DOI 10.1007/s00120-006-1173-9

Online publiziert: 29. August 2006

(c) Springer Medizin Verlag 2006

C. Börgermann $n^{7}$ - M. Sieverding ${ }^{1} \cdot$ P. Fornara ${ }^{2} \cdot$ M. Graefen $^{3} \cdot$ P. Hammerer ${ }^{4}$.

A. Semjonow ${ }^{5} \cdot$ F. Schröder ${ }^{6} \cdot$ H. Rübben ${ }^{7}$

${ }^{1}$ Fachbereich Erziehungswissenschaften und Psychologie, Freie Universität, Berlin

${ }^{2}$ Universitätsklinik und Poliklinik für Urologie, Martin-Luther-Universität,

Halle/Wittenberg

${ }^{3}$ Urologische Klinik, Universitätskrankenhaus Eppendorf, Hamburg

${ }^{4}$ Urologische Klinik, Städtisches Klinikum, Braunschweig

${ }^{5}$ Klinik und Poliklinik für Urologie, Westfälische Wilhelms-Universität, Münster

${ }^{6}$ Abteilung für Urologie, Erasmus Universität, Rotterdam

${ }^{7}$ Klinik und Poliklinik für Urologie, Universitätsklinikum, Essen

\title{
PSA 2010 - Aufbruch in eine neue Ära der Früherkennung des Prostatakarzinom
}

der 70-Jährigen ein latentes Prostatakarzinom aufweisen, befürchtet er eine zunehmende Diagnose klinisch insignifikanter Karzinome [6]. Andere Autoren hingegen erwarten die Abnahme der Korrelation des PSA zum Prostatakarzinom zugunsten des Prostatavolumens, da heutzutage typischerweise kleine organbegrenzte Tumoren gefunden werden. Die Forderung an die Früherkennung ist aber gerade, dass Tumoren nicht zu früh (Übertherapie), aber auch nicht zu spät gefunden werden, um den Patienten eine kurative Therapie anbieten zu können [8].

Zum jetzigen Zeitpunkt bleibt festzuhalten, dass die Effektivität des Screenings beim Prostatakarzinom unbewiesen ist. International besteht Übereinstimmung darüber, dass die Effektivität nur dann bewiesen ist, wenn ein kausaler Zusammenhang zwischen Screening und Verminderung der Sterblichkeit schlüssig mit akzeptabler Lebensqualität und Kosteneffektivität festgestellt werden kann. Zur Evaluation der Effektivität des Screenings werden noch zwei große randomisierte Studien durchgeführt:

- in den USA die PLCO-(Prostate,

Lung, Colorectal and Ovary-)Studie,
- in Europa die ERSPC-(European Randomized Screening for Prostate Cancer-)Studie [9].

Ergebnisse dieser beiden Studien bezüglich des Unterschiedes der Mortalitätsrate des Prostatakarzinoms zwischen dem Screening- und Kontrollarm werden für 2008 erwartet. Folglich kann zum jetzigen Zeitpunkt nicht abschließend entschieden werden, ob populationsbasiertes Screening des Prostatakarzinoms sinnvoll sein wird. Dennoch besteht weitgehender Konsens, dass man heutzutage Männern mit Wunsch nach Früherkennung des Prostatakarzinoms mittels PSA-Test und digital-rektaler Untersuchung diese nicht verweigern sollte [24].

\section{Aktueller Stand der Früherkennung}

Das Prostatakarzinom ist eine Erkrankung des höheren Lebensalters und tritt selten vor dem 50. Lebensjahr auf [19]. In den USA ist das Prostatakarzinom mit $33 \%$ die häufigste neu diagnostizierte Tumorentität und die zweithäufigste krebsbedingte Todesursache (10\%) bei Män- 


\section{Jahre DGU}

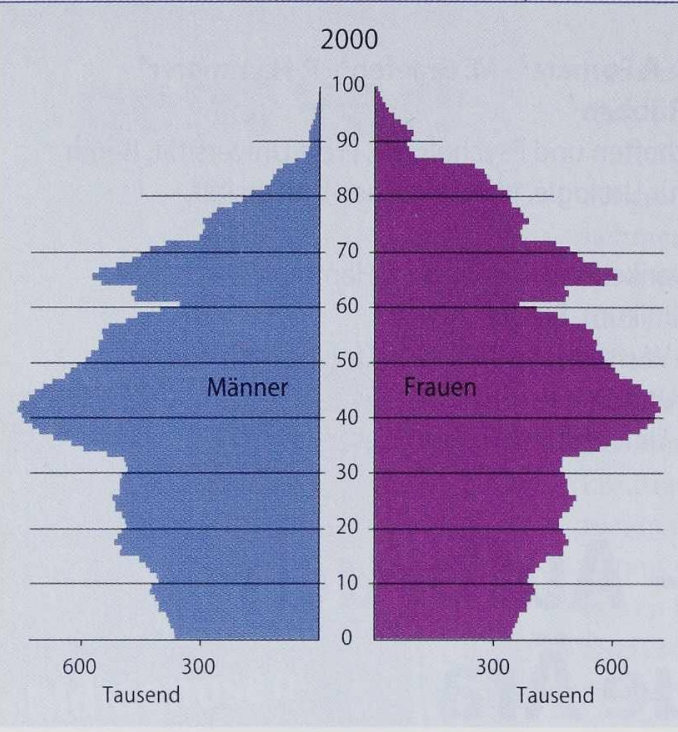

2050

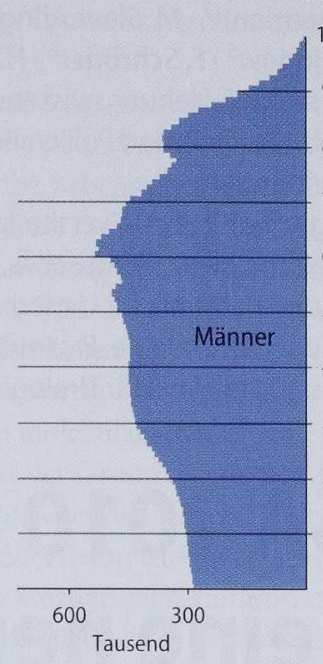

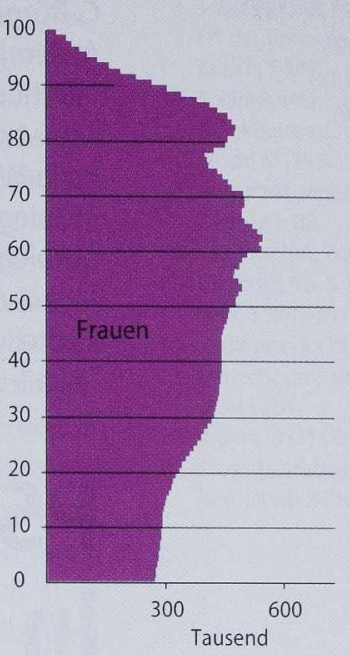

Abb. 1 Bevölkerungsentwicklung von 2000 2050. Im Jahr 2050 wird der Anteil der >60-Jährigen in der Bevölkerung auf 28 Mio. anwachsen nern. Im Jahr 2005 wurden voraussichtlich 232.090 neue Fälle diagnostiziert und 30.350 Patienten werden an ihrem Prostatakarzinom sterben [2].

In Deutschland finden sich vergleichbare Zahlen, jährlich sterben etwa 12.000 Patienten an einem Prostatakarzinom (Statistisches Bundesamt, 2005, http://www.destatis.de/basis/d/gesu/gesutab2o.php). In Zukunft wird die Problematik der Diagnostik und Therapie des Prostatakarzinoms dramatisch zunehmen. Bis zum Jahr 2050 wird der Anteil der $>60$-Jährigen in der Bevölkerung auf $28 \mathrm{Mio}$. anwachsen $(37 \%)$ und somit doppelt so hoch sein wie heutzutage (• Abb. 1).

Die Früherkennung des Prostatakarzinoms muss folgende drei Probleme berücksichtigen:

1. Eine kurative Behandlung des Prostatakarzinoms ist am besten im organbegrenzten Stadium möglich [34]. Da das Prostatakarzinom erst in fortgeschrittenen, zumeist metastasierten Stadien durch eine klinische Symptomatik evident wird, muss die Früherkennung dieses zuvor in lokal begrenzten Stadien detektieren. Andernfalls würden die Patienten trotz aggressiver Therapie in kurativer Intention einen Progress erleiden.

2. Eine Besonderheit dieser Tumorentität ist seine Erscheinungsform als latenter bzw. insignifikanter Tumor, der den Patienten zu Lebzeiten nicht beeinträchtigt. Diese Tumoren sollten nicht detektiert werden, da jede the rapeutische Maßnahme für diese $\mathrm{Pa}$ tienten eine Übertherapie bedeutet.

3. Das Prostatakarzinom hat unbehandelt einen langsamen natürlichen Verlauf, sodass nur Männer mit einer Lebenserwartung von 10-15 Jahren von einer kurativen Therapie profitieren [3].

Somit gehen heute alle Bemühungen in der Karzinomdiagnostik dahin, lokal begrenzte potenziell aggressive Tumoren bei asymptomatischen Männern mit einer Mindestlebenserwartung von 10-15 Jahren zu erkennen. Dabei sollen die latenten Karzinome nicht diagnostiziert werden.

Aktuell wird in Deutschland nach interdisziplinärem Konsens [11] eine jährliche Früherkennungsuntersuchung mittels digital-rektaler Untersuchung und Bestimmung des PSA ab dem 50. Lebensjahr empfohlen, bei positiver Familienanamnese ab dem 45. Lebensjahr. Dabei sollen PSA-Werte ab einem Schwellenwert von $>4 \mathrm{ng} / \mathrm{ml}$ bzw. suspekte rektale Tastbefunde weiter durch eine transrektal sonographisch gesteuerte Prostatabiopsie abgeklärt werden (- Abb. 2).

\section{Problematik}

\section{PSA ist nicht tumorspezifisch}

Da der PSA-Wert ein organspezifischer und kein tumorspezifischer Wert ist, wird seine Serumkonzentration nicht nur durch das Prostatakarzinom bestimmt. Neben dem Malignom kann die PSA-
Konzentration durch eine Vielzahl von Faktoren beeinflusst werden:

1. pharmakologisch (Finasterid, LHRHAnaloga, Antiandrogene),

2. prostatische Erkrankungen (akute/ chronische Prostatitis, BPH, Harnverhaltung),

3. urologische Manipulation (Prostatastanzbiopsie, digital-rektale Untersuchung [21]).

In großen Kollektiven weisen $80-85 \%$ der Patienten einen PSA-Wert $<4 \mathrm{ng} / \mathrm{ml}$ auf, $10-15 \%$ zwischen $4-10 \mathrm{ng} / \mathrm{ml}$ und $2-5 \%$ $>10 \mathrm{ng} / \mathrm{ml}$.

Bei Wertem zwischen 4 und $10 \mathrm{ng} /$ $\mathrm{ml}$ können in der Biopsie in etwa 2025\% der Fälle Prostatakarzinome gefunden werden. Etwa zwei Drittel aller Biopsien in diesem PSA-Bereich sind unauffällig bzw. weisen Charakteristika einer benignen Prostatavergrößerung auf. Bei ca. $11 \%$ kann die Elevation des PSA-Wertes über eine Prostatitis erklärt werden (- Abb.3).

Die Karzinomfindung liegt bei Werten $<4 \mathrm{ng} / \mathrm{ml}$ bei bis zu $10 \%$, zwischen 4 und $10 \mathrm{ng} / \mathrm{ml}$ bei $20-25 \%$ und $>10 \mathrm{ng} / \mathrm{ml}$ steigt dieser Wert parallel zur Organüberschreitung auf $30-50 \%$ an $[16,25]$. Neuere Daten deuten darauf hin, dass die Chance ein Prostatakarzinom kurativ behandeln zu können bis zu einem PSA-Wert von $9 \mathrm{ng} / \mathrm{ml}$ gleich bleibt und sich dann verschlechtert [30], (- Tab. 1, 2). 


\section{Langsamer Krankheitsverlauf}

Obwohl heute die meisten Prostatakarzinome in kurativen Stadien entdeckt werden, zeigt eine skandinavische Studie, dass nach 8 Jahren etwa $15 \%$ aller radikal prostatektomierten und etwa $25 \%$ aller untherapierten Patienten eine Fernmetastasierung aufweisen [5]. Das bedeutet, dass fast $75 \%$ der ausschließlich beobachteten Patienten nach 8 Jahren noch keinen Schaden durch ihr Prostatakarzinom erlitten haben und der Vorteil für die radikale Prostatektomie nur $10 \%$ beträgt. Dies untermauert auf der einen Seite die Befürchtung, dass viele insignifikante Karzinome gefunden und auch therapiert werden, auf der anderen Seite profitieren aber doch 10\% der Patienten von der Therapie.

\section{Insignifikante Karzinome}

Das ungelöste Problem der PSA-basierten Früherkennung ist die hohe Prävalenz an insignifikanten Prostatakarzinomen. Dabei handelt es sich um Karzinome, die ohne einen PSA-Test nie diagnostiziert worden wären. Im Alterssegment > 50 Jahren ist mit meiner Inzidenz von etwa $40 \% \mathrm{zu}$ rechnen. In Zystoprostatektomiepräparaten von Patienten, die aufgrund eines Urothelkarzinoms operiert wurden, findet man in ca. $38 \%$ aller Präparate ein Prostatakarzinom. Diese weisen zu 80\% ein insignifikantes Karzinom nach den Kriterien von Epstein [11] auf. Jemal et al. [12] haben für die USA ein Lebenszeitrisiko für die Diagnose eines Prostatakarzinoms von $17,8 \%$ berechnet. Dem gegenüber werden aber nur 3,5\% der Patienten an einem Prostatakarzinom sterben. Das bedeutet, dass das Lebenszeitrisiko gemessen an Autopsiestudien mehr als 10-mal so hoch ist als das Risiko an einem Prostatakarzinom zu sterben.

\section{Einschränkung der Lebensqualität durch den diagnostischen Prozess}

Bisher wurde die psychische Belastung bzw. die Einschränkung der Lebensqualität durch den diagnostischen Prozess im Rahmen von Prostatakrebsfrüherkennung erst in einigen wenigen Studien dezidiert untersucht $[10,13,18,31]$. Hier gibt es also noch dringenden Forschungsbedarf.
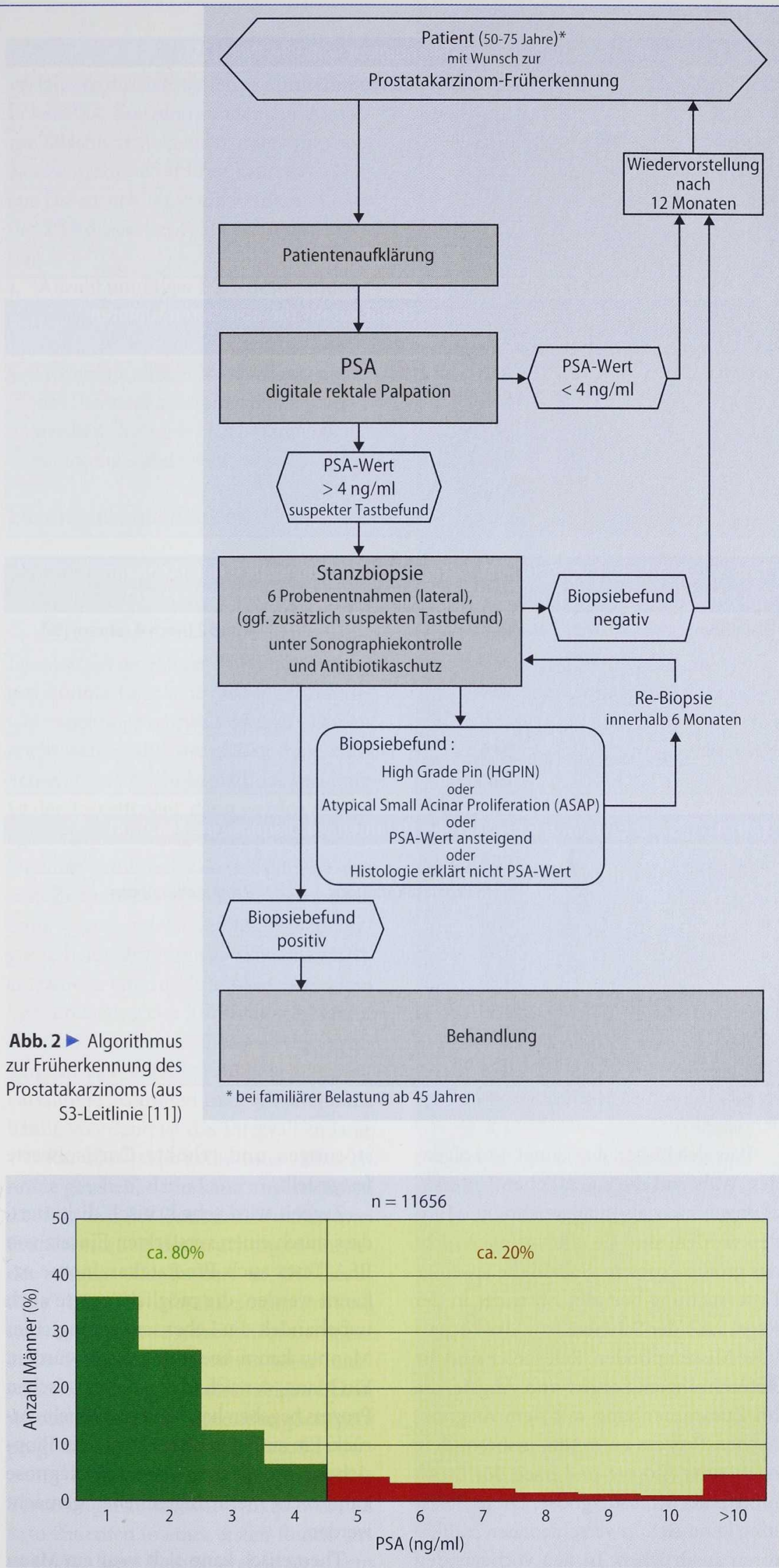

Abb. 3 A Verteilung des PSA-Wertes bei fast 12.000 Männern, die sich zur Früherkennung vorstellten 
Tab. 1 Karzinomfindungsquote in Abhängigkeit vom PSA-Wert [25]

\begin{tabular}{ll}
\hline PSA $[\mathrm{ng} / \mathrm{ml}]$ & Karzinomfindungsrate [\%] \\
$0,0-0,9$ & 0,2 \\
$1,0-1,9$ & 1,3 \\
$2,0-2,9$ & 2,2 \\
$3,0-3,9$ & 6,3 \\
$4,0-9,9$ & 21,7 \\
$>10,0$ & 52,1 \\
\hline
\end{tabular}

Tab. 2 Anteil der organbegrenzten und damit kurativ therapierbaren Tumoren in Abhängigkeit vom PSA-Wert (Carter 1999)

\begin{tabular}{ll} 
PSA [ng/ml] & Anteil $\leq \mathrm{pT} 2[\%]$ \\
$2,5-4,0$ & $78-83$ \\
$4,1-6,0$ & $74-81$ \\
$6,1-8,0$ & $71-78$ \\
$8,1-10,0$ & $67-75$ \\
$>10,0$ & $49-57$ \\
\hline
\end{tabular}

Tab. 3 PSA-Bestimmung in der Prostatakarzinomdiagnostik (Früherkennung des Prostatakarzinoms). Prognostische Faktoren - ERSPC $(n=21.210)$, $[11,25]$

\begin{tabular}{lll} 
Karzinome & Runde 1 [\%] & Rund 2 (nach 4 Jahren) [\%] \\
T1C & 51,0 & 70,8 \\
T2 & 28,6 & 22,1 \\
T3-4 & 19,0 & 7,1 \\
Gleason $\geq 7$ & 34,6 & 22,5 \\
M+ & 0,6 & 0,0 \\
\hline
\end{tabular}

Tab. 4 PSA-Entwicklung innerhalb von 10 Jahren vor Diagnose ( $n=2462)$, [4]

\begin{tabular}{lll} 
Jahr & PSA $[\mathrm{ng} / \mathrm{ml}]$ & \\
& Kein Prostatakarzinom & Prostatakarzinom \\
0 & 1,18 & 2,28 \\
2 & 1,28 & 2,42 \\
4 & 1,36 & 2,55 \\
6 & 1,39 & 3,29 \\
8 & 1,45 & 4,23 \\
10 & 1,49 & 6,37 \\
\hline
\end{tabular}

Was sich bisher abzeichnet, ist Folgendes: Während die eigentlichen Untersuchungen zwar als unangenehm empfunden werden, sind sie offensichtlich nicht das größte Problem. Sobald die jeweilige Untersuchung beendet ist, endet in der Regel auch der Schmerz bzw. das körperliche Missempfinden. Relevanter sind die Befürchtungen, Sorgen und Ängste, die im Zusammenhang mit dem diagnostischen Prozess auftreten, insbesondere nach einer Biopsie und nach der Eröffnung einer Krebsdiagnose. Diese Belastung kann sich in verschiedenen Indikatoren ausdrücken. In den vorliegenden Studien wurden bei den betroffenen Männern u. a. Anstiege von psychischem Stress und Angst, Zunahme von Schlaf- ful waiting"). Er muss dann aber ab diesem Zeitpunkt mit der Gewissheit leben, Krebs zu haben. Oder er entscheidet sich doch für eine radikale Operation oder eine Strahlentherapie und muss sich mit dem Risiko der bekannten Nebenwirkungen von Inkontinenz und Impotenz auseinander setzen. Eine solche Entscheidungssituation ist in hohem MaBe stresserzeugend, und die Lebensqualität nach der Entscheidung für eine radikale Therapieform nimmt umso stärker $a b$, je stärker die Einschränkungen der urologischen und sexuellen Funktionen sind.

Wie Männer mit solchen Entscheidungssituationen umgehen, hängt nicht zuletzt von ihrer Persönlichkeit (z. B. ihrem Angstbewältigungsstil) ab. Es gibt sicher Männer, die trotz aller Nebenwirkungen frühzeitig eine mögliche Prostatakarzinomerkrankung wissen wollen und alles medizinisch Mögliche unternehmen wollen, um ihre Lebenserwartung zu erhöhen. Es gibt andererseits vermutlich Männer, für die eine Krebsdiagnose und -therapie und die mit der Therapie einhergehenden Nebenwirkungen so belastend wären, dass sie lieber das Risiko eingehen, ein mögliches Prostatakarzinom erst (zu) spät zu entdecken. Von daher kann es gerade bei der Prostatakarzinomfrüherkennung keine generelle Empfehlung geben, die für jeden Einzelfall zutrifft.

Für die PSA-basierte Früherkennung kann aus dem oben dargestellten folgendes Zahlenbeispiel abgeleitet werden: Von 1 Mio. Männern, die sich einer PSABestimmung unterziehen, haben 200.000 einen Wert $>4 \mathrm{ng} / \mathrm{ml}$ und sollen nach den bestehenden Leitlinien durch eine Prostatabiopsie weiter abgeklärt werden. Hier erwartet man eine Fallfindungsquote von etwa $25 \%$, also 50.000 diagnostizierte Prostatakarzinome. Selbst unter der Annahme, dass diese Patienten alle erfolgreich kurativ behandelbar wären, würden nur $25 \%$ also 12.000 Männer gegenüber keiner Therapie profitieren. Anders ausgedrückt würde nur gut $1 \%$ aller Männer von der Durchführung des PSA-Tests profitieren (- Abb.4). 


$\begin{array}{ll}\text { PSA-Test } & \text { Patienten zur Früherkennung } \\ 200.000 & \text { Patienten mit PSA }>4 \mathrm{ng} / \mathrm{ml} \\ \text { Biopsie } & \\ 50.000 & \text { Prostatakarzinome } \\ 12.500 & \text { Pad. Prostatektomie } \\ & \text { Patienten profitieren von } \\ & \text { kurativer Therapie }\end{array}$

Abb. $4 \Delta$ Bei der heute durchgeführten Früherkennung profitieren nur gut $1 \%$ aller untersuchten Männer

\section{Biologische Schwankungen des PSA-Wertes}

Für die Beurteilung einer intraindividuellen PSA-Dynamik ist die Kenntnis der biologischen Variabilität erforderlich. Diese ist höher als allgemein erwartet und beträgt nach einer Literaturübersicht ca. 20\% [29]. Zusammen mit der analytischen Variabilität der PSA-Messverfahren von ca. 5\% bedeutet dies, dass bei einer einmaligen Messung eines PSA-Wertes von $3,3 \mathrm{ng} / \mathrm{ml}$, die Obergrenze des 95\%-Konfidenzintervalls bei $4,4 \mathrm{ng} / \mathrm{ml}$ liegt. Ergeben drei in zeitlichem Abstand durchgeführte PSA-Messungen einen Mittelwert von $3,3 \mathrm{ng} / \mathrm{ml}$, so reduziert sich die Obergrenze des $95 \%$-Konfidenzintervalls auf $3,9 \mathrm{ng} / \mathrm{ml}$.

\section{Fehlende Standardisierung von PSA-Messverfahren}

Derzeit kann kein für alle PSA-Messverfahren gültiger, einheitlicher Grenzwert für die Entscheidung zu einer Prostatabiopsie angegeben werden [28]. Die von verschiedenen PSA-Messverfahren in identischen Proben gefundenen PSAKonzentrationen können sich klinisch relevant voneinander unterscheiden. Daher sind für jedes PSA-Messverfahren nur die mit ihm selbst bestimmten Entscheidungsgrenzen klinisch sinnvoll einsetzbar $[26,32]$. So finden z. B. „standardisierte" PSA-Messverfahren in einer Probe 3,X $\mathrm{ng} / \mathrm{ml}$, in den Messverfahren, die sich in ihrer Kalibrierung an den Messverfahren der Fa. Hybritech (Beckman Coulter) orientieren jedoch 4,0 ng/ml PSA.

Jedes PSA-Messverfahren hat eine individuelle PSA-Entscheidungsgrenze, die von $4 \mathrm{ng} / \mathrm{ml}$ abweichen kann. In der Verlaufsbeobachtung von PSA-Werten muss ein Wechsel des Verfahrens berücksichtigt werden, da sonst der Wechsel des Messverfahrens durch Erkennung unterschiedlicher PSA-Konzentrationen zur Annahme fälschlich steigender oder sinkender Konzentrationen führen kann [27]. Daraus lassen sich folgende Kritikpunkte an der PSA-basierten Früherkennung ableiten:

1. Anzahl unnötiger PSA-Bestimmungen,

2. Anzahl unnötiger Biopsien,

3. Anzahl unnötiger Behandlungen, da das Prostatakarzinom trotzdem progredient wird oder auch ohne Behandlung stabil bleibt.

\section{Lösungsmöglichkeiten}

\section{Screeningintervall individuell gestalten}

Die Anzahl unnötiger PSA-Bestimmungen könnte über eine Verlängerung des Untersuchungsintervalls von zzt. 1 Jahr erreicht werden. Bei der Anwendung eines Screeningtests erhöht sich die Inzidenz. Ist der Test effizient, dann werden die Tumoren früher, $d$. h. in einem präklinischen Stadium gefunden. Die gewonnene Zeit vom Zeitpunkt des positiven Tests bis zur ohne Test zu erwarteten klinischen Diagnose heißt „leadtime“. Nach einer effizienten Screeningrunde kommt es zu einer Verminderung der klinischen Inzidenz. Karzinome, die zwischen zwei Testrunden klinisch auffallen heißen Intervallkarzinome. Kommen Intervallkarzinome häufig vor, dann ist das Intervall zu lang, werden überhaupt keine Intervallkarzinome gesehen, dann kann angenommen werden, dass der Zeitraum zwischen zwei Früherkennungsuntersuchungen entweder adäquat oder zu kurz ist. Die „leadtime" beim Prostatakarzinom ist lang und beträgt etwa 5-10 Jahre. Bei jährlicher Früherkennung treten keine Intervallkarzinome auf. Generell zeigen sich in wiederholten Screeningrunden günstigere prognostische Faktoren (- Tab. 3).

Im Rahmen der ERSPC-Studie im Screeningarm in Rotterdam wurden 8350 Patienten in einer ersten Runde untersucht. Eine Biopsie wurde ab einem PSA-Schwellenwert von $4 \mathrm{ng} / \mathrm{ml}$, im späteren Verlauf der Studie ab 3 ng/ml (PSAMessverfahren Beckman-Coulter Hybri-

\section{Sexualstörungen}

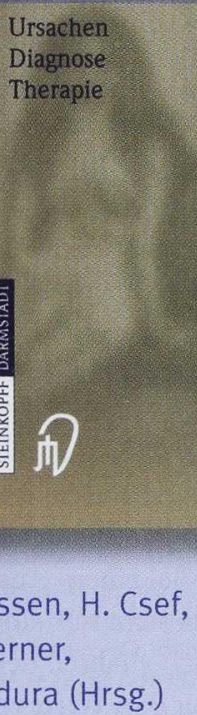

Sexualstörungen

\section{Ursachen - Diagnose -}

\section{Therapie}

2006. 192 Seiten. Geb.

EUR 44,95*

ISBN 3-7985-1547-6

Sexualität und ihre Störungen haben in den letzten Jahrzehnten einen tief greifenden normativen Wandel erfahren. Immer mehr Menschen wünschen sich Beratung und therapeutische Hilfe.

"Sexualstörungen“ bietet Diagnostik, Beratung, Behandlung von sexuellen Störungen in allen Lebensabschnitten.

\section{Weitere Themen:}

- Sexueller Missbrauch

- Geschlechtsspezifische Dysfunktionen

- Fertilitätsstörungen

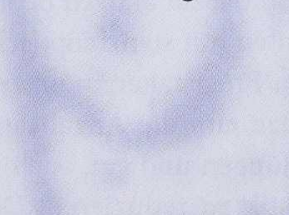

Steinkopff Darmstadt c/o Springer Distribution Center Kundenservice · Haberstraße 7 69126 Heidelberg/Germany Fax: +49-6221-345-4229 E-Mail: SDC.bookorder@springer.com www.steinkopff.springer.de 
tech) oder bei einem auffälligen Tastbefund empfohlen. Dabei wurden 412 Prostatakarzinome diagnostiziert. In einem Intervall von 48 Monaten fielen 18 Intervallkarzinome auf, alle im Tumorstadium $\mathrm{T}_{1}$ bzw. T2 und damit kurativ therapierbar [33]. Eine aktuelle Arbeit [23] zeigt, dass sogar ein Intervall von 8 Jahren noch effektiv sein kann. Von $1703 \mathrm{~Pa}$ tienten mit einem initialen PSA-Wert von $<1 \mathrm{ng} / \mathrm{ml}$ wurden innerhalb von 8 Jahren nach dem 1 . Screening 8 Prostatakarzinome entdeckt.

Ein optimales Intervall für die Früherkennung durch PSA-Test kann zum jetzigen Zeitpunkt noch nicht festgelegt werden und hängt entscheidend vom Ausgangswert ab. Bei einem Ausgangs-PSA von $<1 \mathrm{ng} / \mathrm{ml}$ besteht nur ein Risiko von 0,01\%, dass nach 4 Jahren der PSA-Wert $>3 \mathrm{ng} / \mathrm{ml}$ ist. Für PSA Werte von $<2 \mathrm{ng} / \mathrm{ml}$ steigt das Risiko auf knapp 1\%. In Anbetracht der Datenlage erscheint daher eine jährliche Untersuchung für Männer mit derart niedrigen PSA-Konzentrationen nicht sinnvoll. Eine Verlängerung des Intervalls würde über weniger durchgeführte PSA-Bestimmungen und Biopsien neben der Kosteneinsparung insbesondere zur Vermeidung einer unnötigen Beein trächtigung der Lebensqualität führen.

Eine Anpassung der Früherkennungsfrequenz an den PSA-Verlauf und die Möglichkeit des Einzelnen, die Frequenz der Früherkennungsuntersuchung mitzubestimmen, sind realistische zukünftige Möglichkeiten. Starr festgelegte, für alle Probanden gültige Intervalle sollten nicht mehr angewandt werden. Stattdessen empfiehlt sich eine individuelle $\mathrm{Ge}$ staltung der Intervalle, die sich zum einen am letzten PSA-Wert und zum anderen am individuellen PSA-Verlauf orientiert.

\section{Risikoabschätzung}

Eine Studie zur aktiven Beobachtung von Patienten mit stanzbioptisch nachgewiesenem Prostatakarzinom zeigt mögliche Ansätze, um die Anzahl unnötiger Behandlungen und ggf. auch unnötige Diagnostik zu reduzieren. Dabei wurden 299 Patienten mit gesicherter Diagnose eines Prostatakarzinoms mit niedrigem Risiko nicht therapiert sondern aktiv beobachtet. Die Patienten waren $>70$ Jah- re, der Gleason-Score betrug <7, das PSA $<15 \mathrm{ng} / \mathrm{ml}$ und das klinische Tumorstadium maximal cT2b.

Ein Progress wurde u. a. über eine PSAVerdopplungszeit von $<2$ Jahren definiert. Nach einer mittleren Beobachtungszeit von 55 Monaten betrug das krankheitsspezifische Überleben $99 \%$ und 60\% der Patienten zeigten keinen Progress und wurden weiter aktiv nachbeobachtet [15].

Diese Ergebnisse veranlassten den $\mathrm{Au}$ tor, eine neue Studie zu initiieren. Hierbei wurde der Progress über eine PSAVerdopplungszeit von $<3$ Jahren definiert, um eine größere Sicherheit für die Patienten zu erreichen. Auf die Früherkennung angewendet, könnte dementsprechend die Dynamik des PSA-Wertes helfen, Biopsien zu sparen und Lebensqualität zu verbessern. Die oben genannten $60 \%$ der Patienten, die in der Beobachtungsgruppe verblieben, hätten, wenn man als Kriterium für die Stanzbiopsie eine PSA-Verdopplungszeit von <2 Jahren fordern würde, keine Biopsie erhalten sie würden deshalb Ihre Diagnose nicht kennen und damit in der Lebensqualität profitieren. Auch andere Autoren kommen zu dem Schluss, dass die „active surveillance " für etwa zwei Drittel aller $\mathrm{Pa}$ tienten mit einem lokal begrenzten Prostatakarzinom möglich ist, ohne deren Lebenserwartung einzuschränken [17].

Die Arbeitsgruppen von Carter und Potter beschrieben schon im Jahr 2000 [20], dass bei einer PSA-Verlaufsbeobachtung über 8-26 Jahre 95\% aller Männer ohne Prostatakarzinom einen PSAAnstieg von $<0,75 \mathrm{ng} / \mathrm{ml} / \mathrm{Jahr}$ haben und 70\% aller Patienten mit Prostatakarzinom eine Anstiegsgeschwindigkeit von $>0,75 \mathrm{ng} / \mathrm{ml} / \mathrm{Jahr}$ aufweisen. In einer aktuellen Arbeit von Berger et al. [4] mit einer maximalen Beobachtungszeit von 10 Jahren hatten Männer mit späterer Prostatakarzinomdiagnose eine mittlere PSAAnstiegsgeschwindigkeit von $0,4 \mathrm{ng} / \mathrm{ml} /$ Jahr gegenüber von $0,03 \mathrm{ng} / \mathrm{ml} / \mathrm{Jahr}$ für Männer ohne Prostatakarzinomdiagnose. Über einen Beobachtungszeitraum von 4 Jahren konnten Raaijmakers et al. [22] in der ERSPC-Studie allerdings keinen klinisch relevanten Vorteil für die PSAAnstiegsgeschwindigkeit oder -Verdopplungszeit finden (- Tab.4).
Der Einsatz der PSA-Dynamik (Veränderung über die Zeit) kann einen Anhalt dafür geben, wie aggressiv ein Prostatakarzinom ist. Der Einsatz der PSADynamik könnte also schon vor der invasiven Diagnostik helfen, zwischen den Patienten zu unterscheiden, die frühzeitig therapiert werden sollten bzw. denjenigen, die voraussichtlich ein klinisch insignifikantes Karzinom aufweisen. Obwohl das Konzept der PSA-Anstiegsgeschwindigkeit schon seit vielen Jahren verfolgt wird, ist es noch nicht möglich eine abschlieBende Empfehlung zu geben.

\section{Mögliche Zukunft der PSA- basierten Früherkennung}

1. Beginn mit 40 Jahren: In diesem Altersbereich erwarten wir bei dem größten Teil der Männer einen niedrigen PSA-Wert ( $<2 \mathrm{ng} / \mathrm{ml})$. Die Inzidenz des Prostatakarzinoms steigt deutlich beginnend mit 50 Jahren, sodass man einen ausreichend langen Zeitraum (mindestens 10 Jahre) hat, um die PSA-Dynamik zu beurteilen.

2. Ende bei einer Lebenserwartung von $<10$ Jahren: In diesem Punkt besteht ein breiter Konsens. Die Vorhersage der Lebenserwatung ist aber schwierig. Eine Orientierung bieten für dieses Patientenkollektiv die Nomogramme von Albertsen et al. [1].

3. Intervall alle 2 Jahre für Patienten ohne Risikofaktoren (PSA $<2$ ng/ml): Auch wenn das ideale Screeninginterval noch unklar ist, so sprechen die Daten dafür, dass Patienten mit niedrigem PSA und negativem Tastbefund nicht gefährdet werden, wenn das Intervall verlängert wird. Dies würde immerhin etwa zwei Drittel aller Patienten betreffen.

4. Biopsieindikation bei einer PSA-Anstiegsgeschwindigkeit von $>0,5 \mathrm{ng} /$ $\mathrm{ml} / \mathrm{Jahr}$ (Abschaffung des starren Schwellenwertes): Die Angegebene PSA-Anstiegsgeschwindigkeit ist nur ein willkürlich festgelegter Wert, der sich nur auf die Patienten bezieht, die mit niedrigen PSA-Werten ihr Screening begonnen haben. Es werden Studien folgen müssen, um diesen Schwellenwert näher zu definieren. 
Eine Anpassung der Früherkennungsfrequenz an den PSA-Verlauf und die Möglichkeit des Einzelnen, die Frequenz der Früherkennungsuntersuchung mitzubestimmen, sind realistische zukünftige Möglichkeiten. Starr festgelegte, für alle Probanden gültige Intervalle sollten nicht mehr angewandt werden. Es ist noch nicht möglich für das Konzept der PSAAnstiegsgeschwindigkeit eine abschlieBende Empfehlung zu geben.

\section{Korrespondierender Autor \\ Dr. C. Börgermann}

Klinik und Poliklinik für Urologie

Universitätsklinikum

Hufelandstraße 55

45122 Essen

christof.boergermann@uk-essen.de

Interessenkonflikt. Es besteht kein Interessenkonflikt. Der korrespondierende Autor versichert, dass keine Verbindungen mit einer Firma, deren Produkt in dem Artikel genannt ist, oder einer Firma, die ein Konkurrenzprodukt vertreibt, bestehen. Die Präsentation des Themas ist unabhängig und die Darstellung der Inhalte produktneutral.

\section{Literatur}

1. Albertsen PC, Fryback DG, Storer BE et al. (1996) The impact of co-morbidity on life expectancy among men with localized prostate cancer. J Urol 156(1): 127-132

2. American Cancer Society (2005) Cancer Facts \& Figures (http://www.cancer.org/downloads/STT/ CAFF2005f4PWSecured.pdf

3. Aus G, Hugosson J, Norlen L (1995) Long-term survival and mortality in prostate cancer treated with noncurative intent. J Urol 154(2 Pt 1): 460-465

4. Berger AP, Deibl M, Steiner Het al. (2005) Longitudinal PSA changes in men with and without prostate cancer: assessment of prostate cancer risk. Prostate 64(3): 240-245

5. Bill-Axelson A, Holmberg L, Ruutu M et al. (2005) Radical prostatectomy versus watchful waiting in early prostate cancer. N Engl J Med 352(19): 19771984

6. Brawer MK, Chetner MP (1997) Campell's Urology. In: Walsh PC, Retik A, Stamey TA, Vaughan EJ (eds) Saunders, Philadelphia, pp 2506-2518

7. Catalona WJ, Richie JP, Ahmann FR et al. (1994) Comparison of digital rectal examination and serum prostate specific antigen in the early detection of prostate cancer: results of a multicenter clinical trial of 6,630 men. J Urol 151(5): 1283-1290

8. Catalona WJ, Loeb S (2005) The PSA era is not over for prostate cancer. Eur Urol 48(4): 541-545

9. De Koning HJ, Liem MK, Baan CA et al. (2002) Prostate cancer mortality reduction by screening: power and time frame with complete enrollment in the European Randomised Screening for Prostate Cancer (ERSPC) trial. Int J Cancer 98(2): 268-273

10. Essink-Bot ML, De Koning HJ, Nijs HG et al. (1998) Short-term effects of population-based screening for prostate cancer on health-related quality of life. J Natl Cancer Inst 90(12): 925-931
11. Fachgesellschaften (2002) Arbeitsgemeinschaft wissenschaftlich medizinischer Fachgesellschaften. S3-Leitlinie: PSA-Bestimmung in der Prostatakarzinomdiagnostik (Früherkennung des Prostatakarzinoms)http://www.awmf-online.de

12. Gosselaar C, Roobol MJ, Schroder FH (2005) Prevalence and characteristics of screen-detected prostate carcinomas at low prostate-specific antigen levels: aggressive or insignificant? BJU Int 95(2): 231-237

13. Gustafsson O, Theorell T, Norming U et al. (1995) Psychological reactions in men screened for prostate cancer. Br J Urol 75(5): 631-636

14. Hoedemaeker RF, Rietbergen JB, Kranse R et al. (2000) Histopathological prostate cancer characteristics at radical prostatectomy after population based screening. J Urol 164(2): 411-415

15. Klotz L (2004) Active surveillance with selective delayed intervention: using natural history to guide treatment in good risk prostate cancer. J Urol 172(5 Pt 2): 48-50

16. Luboldt HJ, Altwein JE, Bichler KH et al. (1999) Early recognition of prostate carcinoma. Initial results of a prospective multicenter study in Germany. Project Group for Early Detection DGU-BDU Laboratory diagnosis Professional Circle. Urologe A 38(2): 114-123

17. Parker C (2004) Active surveillance: towards a new paradigm in the management of early prostate cancer. Lancet Oncol 5(2): 101-106

18. Perczek RE, Burke MA, Carver CS et al. (2002) Facing a prostate cancer diagnosis: who is at risk for increased distress? Cancer 94(11): 2923-2929

19. Pienta KJ (1997) Etiology, epidemiology and prevention of carcinoma of the prostate. In: Walsh PC, Retik A, Stamey TA, Vaughan EJ, (eds) Campell's Urology. Saunders, Philadelphia, pp 2489-2496

20. Potter SR, Carter HB (2000) The role of prostatespecific antigen velocity in prostate cancer early detection. Curr Urol Rep 1(1): 15-19

21. Price CP, Allard J, Davies G et al. (2001) Pre- and post-analytical factors that may influence use of serum prostate specific antigen and its isoforms in a screening programme for prostate cancer. Ann Clin Biochem 38(Pt 3): 188-216

22. Raaijmakers R, Wildhagen MF, Ito K et al. (2004) Prostate-specific antigen change in the European Randomized Study of Screening for Prostate Cancer, section Rotterdam. Urology 63(2): 316-320

23. Roobol MJ, Roobol DW, Schroder FH (2005) Is additional testing necessary in men with prostatespecific antigen levels of $1.0 \mathrm{ng} / \mathrm{mL}$ or less in a population-based screening setting? (ERSPC, section Rotterdam). Urology 65(2): 343-346

24. Schmid HP, Riesen W, Prikler L (2004) Update on screening for prostate cancer with prostate-specific antigen. Crit Rev Oncol Hematol 50(1): 71-78

25. Schröder FH, Van DC-K, De Koning HJ et al. (2000) Prostate cancer detection at low prostate specific antigen. J Urol 163(3): 806-812

26. Semjonow A, De Angelis G, Oberpenning F et al. (2000) The clinical impact of different assays for prostate specific antigen. BJU Int 86(5): 590-597

27. Semjonow A, Schmid HP (2002) The rise and fall of PSA: clinical implications of prostate specific antigen kinetics. Urol Res 30(2): 85-88

28. Semjonow A (2006) Unterschiede zwischen PSABestimmungsverfahren. In: Fornara P, Semjonow A (Hrsg) PSA: Der Weg zum Befund - Präanalytik und Analytik des prostataspezifischen Antigens. Zuckschwerdt, München
29. Soletormos G, Semjonow A, Sibley PE et al. (2005) Biological variation of total prostate-specific antigen: a survey of published estimates and consequences for clinical practice. Clin Chem 51(8): 1342-1351

30. Stamey TA, Caldwell M, MCNeal JE et al. (2004) The prostate specific antigen era in the United States is over for prostate cancer: what happened in the last 20 years? J Urol 172(4 Pt 1): 1297-1301

31. Steginga SK, Occhipinti S (2006) Dispositional optimism as a predictor of men's decision-related distress after localized prostate cancer. Health Psychol 25(2): 135-143

32. Stephan C, Klaas M, Muller C et al. (2006) Interchangeability of measurements of total and free prostate-specific antigen in serum with 5 frequently used assay combinations: an update. Clin Chem 52(1): 59-64

33. van der Cruijsen-Koeter IW, van der Kwast TH, Schroder FH (2003) Interval carcinomas in the European Randomized Study of Screening for Prostate Cancer (ERSPC)-Rotterdam. J Natl Cancer Inst 95(19): 1462-1466

34. Walsh PC, Partin AW, Epstein JI (1994) Cancer control and quality of life following anatomical radical retropubic prostatectomy: results at 10 years. J Urol 1152(5 Pt 2): 1831-1836 